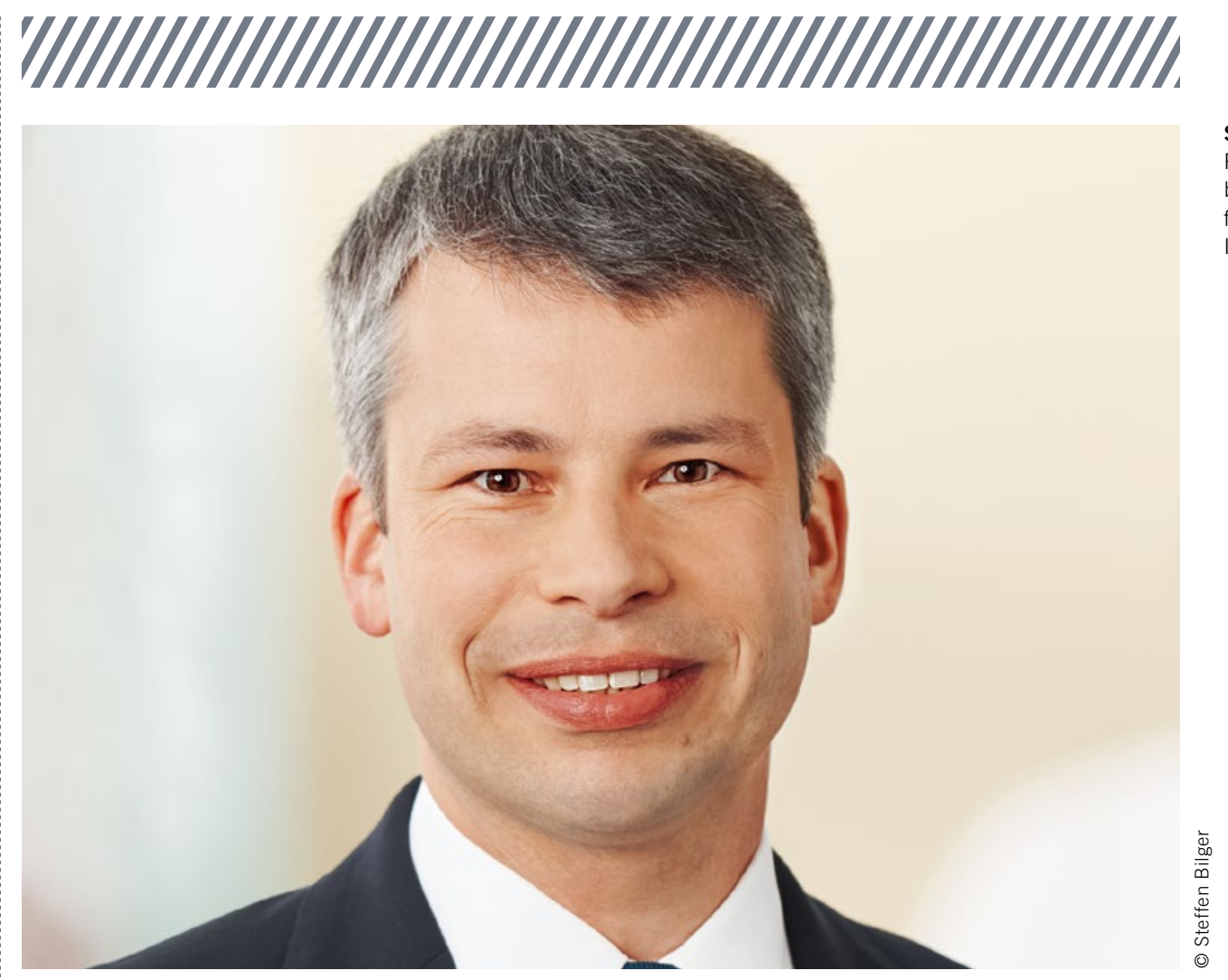

\title{
Zukunftsorientierte digitale Mobilitätspolitik
}

Mobilität ist eine Grundlage für individuelle Freiheit, für gesellschaftlichen Wohlstand, für wirtschaftliches Wachstum und Arbeitsplätze in Städten und Regionen. Als Hochtechnologieland, als Export- und als Transitnation nutzen wir die Digitalisierung für die Entwicklung eines zukunftsfähigen Verkehrssystems. Mobilität muss sicher, sauber, effizient, barrierefrei, nachhaltig und bezahlbar sein und bleiben.

Deshalb setzt sich die Bundesregierung unter anderem weiter verstärkt für die Schaffung optimaler Rahmenbedingungen bei der Einführung von Systemen des automatisierten und vernetzten Fahrens (AVF) in den Regelbetrieb des Straßenverkehrs ein.

Auf Grundlage der in 2015 verabschiedeten „Strategie automatisiertes und vernetztes Fahren - Leitanbieter bleiben, Leitmarkt werden, Regelbetrieb einleiten" hat sich Deutschland als Vorreiter und internationaler Impulsgeber beim AVF etabliert. Diese Position wollen wir halten und mit konkreten Maßnahmen weiter ausbauen.

Im Straßenverkehrsgesetz haben wir als erstes Land weltweit bereits grundlegende Regelungen und Voraussetzungen für ein Abwenden vom Verkehrsgeschehen und der Fahrzeugsteuerung bei der Fahrzeugführung mittels automatisierter Fahrfunktionen geschaffen. Nun entwickeln wir einen Rechtsrahmen für das autonome Fahren in speziellen Anwendungsfällen.
Steffen Bilger

Parlamentarischer Staatssekretär beim Bundesminister ür Verkehr und digitale Infrastruktur
Der Bund unterstützt weiter die Forschung zum AVF und das Erproben von Innovationen auf digitalen Testfeldern im Realverkehr. Im Blick haben wir dabei sowohl den Individualals auch den öffentlichen Personen- und den Güterverkehr. Wir wollen bedarfsgerechte Mobilitätsangebote schaffen. Daher werden wir im Dialog mit Wirtschaft, Wissenschaft, Ländern und Kommunen neue Mobilitätskonzepte entwickeln, die insbesondere eine verbesserte Verknüpfung der verschiedenen Verkehrsträger beinhalten. Als Grundlage für das vernetzte Fahren soll die digitale Infrastruktur verbessert werden. Ebenso intensivieren wir den Ausbau der Verkehrstechnik durch kooperative intelligente Verkehrssysteme. Hohe Priorität haben Aktivitäten bei internationaler Standardisierung, Cybersicherheit, Datenschutz und -nutzung.

Der gesellschaftliche Dialog zur Zukunft der Mobilität ist uns ein wichtiges Anliegen. Eine unabhängige Ethik-Kommission hat Regeln entwickelt, die den Rahmen für eine Zulassung automatisierter Fahrsysteme zum Straßenverkehr unter Berücksichtigung von Sicherheit, menschlicher Würde, persönlicher Entscheidungsfreiheit und Datenautonomie setzen. Den daraus abgeleiteten Maßnahmenplan der Bundesregierung werden wir umsetzen. Mit den Bürgern sowie den Kommunen müssen wir vor Ort die Chancen und Risiken der neuen Technologien erörtern. 


\section{Springer Vieweg}

F. Wolf

\section{Fahrzeuginformatik}

Eine Einführung in die Softwareund Elektronikentwicklung aus der

Praxis der Automobilindustrie

2018. XXVII, 314 S. 168 Abb., 95 Abb. in Farbe.

Book + eBook. Brosch.

$€(D) 34,99|€(A) 35,83|$ CHF 35.50

ISBN 978-3-658-21223-0

$€$ (D) 26,99 | CHF 28.00

ISBN 978-3-658-21224-7 (eBook)

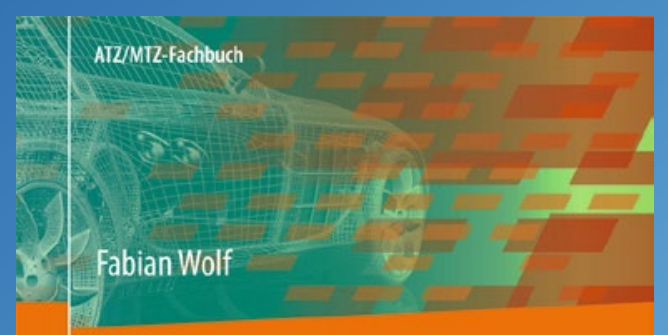

\section{Fahrzeuginformatik}

Eine Einführung in die Software- und Elektronikentwicklung aus der Praxis der Automobilindustrie

\section{Allgemeine Zusammenfassung und Übersicht über Entwicklung und Prozesse für Elektronik und Software im Fahrzeug}

- Fachliche Basis für Entscheider außerhalb der Automobilindustrie

- Diskussionsbasis für das nachhaltige und fundierte Vorantreiben des digitalen Wandels

Dieses Fach- und Lehrbuch enthält die heutigen Grundlagen und Sicht der Praxis zu Elektronik und Software im Fahrzeug. Es dient damit als Basis für die Umsetzung neuer Trends, wissenschaftliche Arbeiten und ökonomische Entscheidungen. Elektronikarchitektur, Softwareentwicklung und Test sowie die zugehörigen Prozesse für zulassungsrelevante Systeme werden vorgestellt. Die „Fahrzeuginformatik“ spielt als Schnittstelle zwischen Produktvision und deren konkreter Umsetzung eine Schlüsselrolle in der Elektromobilität. Damit ist dieser Begriff rein sprachlich das deutsche Pendant zum „Automotive Software Engineering" und definiert darüber hinaus ganzheitlich eine neue Disziplin in der Entwicklung der Fahrzeuggenerationen von morge $\mathrm{n}$. Die Zielgruppen sind in erster Linie angehende Fachkräfte der Mechatronik, Studierende und Berufseinsteiger im Bereich der Fahrzeugelektronik und Fahrzeuginformatik sowie sämtliche Mitarbeiter der Automobilindustrie, die sich auf den anstehenden Wandel nachhaltig vorbereiten wollen, um Entscheidungen auf der Basis von Fakten zu treffen. Damit empfiehlt sich [...]

$€(D)$ sind gebundene Ladenpreise in Deutschland und enthalten $7 \%$ für Printprodukte bzw. $19 \%$ MwSt. für elektronische Produkte. $€(A)$ sind gebundene Ladenpreise in Österreich und enthalten $10 \%$ für Printprodukte bzw. $20 \%$ MwSt. für elektronische Produkte. Die mit* gekennzeichneten Preise sind unverbindliche Preisempfehlungen und enthalten die landesübliche MwSt. Preisänderungen und Irrtümer vorbehalten. 